\title{
An experimental analysis of a stand-alone standing wave thermoacoustic refrigerator
}

\author{
Giulio Allesina
}

Received: 1 October 2013 / Accepted: 9 January 2014/Published online: 12 March 2014

(c) The Author(s) 2014. This article is published with open access at Springerlink.com

\begin{abstract}
In the last years, the thermoacoustic phenomena had risen the scientific community interest due to its implications in thermodynamics, heat transfer and acoustic matters, that make possible the conversion between sound and heat. This work aimed at exploring the Russell-Weibull refrigerator, analyzing the behavior of the different parts to achieve a good level of thermal separation, together with the capability of working standalone. Special attention was paid to all those parts that need to be designed properly with explanation of the physics that ruled them, such as the sound generator, the woofer box, the stack and the tube insulation. During the second part of the work, the results obtained are discussed, consistently showing that, if some expedients are adopted, this simple refrigerator can achieve a temperature difference of $24^{\circ} \mathrm{C}$. The design choices concerning materials and geometries had great influence on the device behavior.
\end{abstract}

Keywords Thermoacoustic - Standing wave .

Refrigerator

\section{Introduction}

Eleven years have elapsed since the work by Russell and Weibull on tabletop refrigerator has been published [1]. During this time span, thermoacoustic devices have made much progress, improving technologies and efficiencies $[14,15,18]$. Despite this progress, the Russell-Weibull

\section{G. Allesina $(\bowtie)$}

BEELab, Department of Engineering 'Enzo Ferrari', University of Modena and Reggio Emilia, Via Vignolese 905/b, 41125 Modena, Italy

e-mail: giulio.allesina@unimore.it refrigerator still remains the simplest way to demonstrate the charm of the thermoacoustic refrigeration and the physics phenomena on which it rests. In the last two decades, the thermoacoustic technology has drawn the attention of many research groups looking for a different way to obtain cooling [19]. This attention resulted in the development of several refrigerators, from prototypes to industrial applications [20, 21]. The fundamental advantages related to this technology are: simplicity, compactness and reliability, absence of moving parts (i.e. neither wear nor lubrication), inert and environmental friendly working fluid. Moreover, the refrigerating power is adjustable as a function of the sound amplitude (the compressor in common refrigerators works just in on-off mode) [2]. However, since the first Hofler's thermoacoustic cooling engine was built [3], these devices have dealt with some limitations, such as low thermal power, low efficiency, the need for an accurate tuning of the system, noise pollution [4, 18]. A great deal of effort was made in different directions to alleviate these problems. The proposed solutions resulted in the realization of traveling wave devices, complex geometry resonators, multiple speaker devices, etc. [4-6, 15, 17]. These innovations were responsible for the development of thermoacoustic devices ranging from micro-cooling devices up to 500 GPD acoustic gas liquefiers [7, 8, 15]. Different solutions allowed to fix or restrain the weak points of this technology, but at this stage more progress is needed to compete efficiently against common refrigeration plants.

While it is relatively easy to create simple working devices, it is however quite hard to produce efficient refrigerators. Although simple standing-wave refrigerators may seem obsolete compared with such a technically complex outlook, they remain a starting point for the development of more complex devices and test potential improvements. 
The aim of this work is to explore the experimental feasibility of a stand-alone thermoacoustic refrigerator. In order to design a stand-alone device, the Russell-Weibull refrigerator was used as basis [1]. The T-A refrigerator was then modified from the original design, furthermore an oscillator circuit for the sound generation was implemented. Furthermore, the woofer box design was investigated with particular focus on methods to increase the speaker efficiency. Finally, the apparatus was tested with different stacks, one built as instructed by Russell and Weibull, one built with parallel glass slides.

The adopted solutions have contributed to the production of a stand-alone device. The temperature difference was monitored for all the tests and it resulted consistent with the results of Russell-Weibull, despite the modifications.

\section{Method}

The thermoacoustic refrigerator discussed here was designed on the basic scheme of a standing-wave $t$-a refrigerator [2]. A sketch of the experimental apparatus is displayed in Fig. 1 where, in the left image, the three fundamental parts of the apparatus are sketched: (a) is the loudspeaker that produces a sound (pressure wave) at the resonance frequency of the resonator tube (b), in which a standing wave is generated (one end is closed, the other end is connected to the loudspeaker). (c) Is the stack, i.e., an array of parallel channels. Figure $1 \mathrm{~b}$ shows the refrigerator discussed in this paper. It is an improvement of the basic scheme of Fig. 1a. The additional parts are: (d) an aluminum cork used to seal the tube and disperse heat, (e) a cover sheet set on the woofer in which the resonator is plugged, (f) the woofer box used to contain the sound emission, ( $g$ ) the oscillator circuit used to generate a sinusoidal signal amplified by the power amplifier (h). With these improvements the device is able to work as a stand-alone apparatus.

For all the experiments, a $160 \mathrm{~mm} 60 \mathrm{~W}$ RMS-power woofer was used. The woofer was powered by a simple 100 W RMS Darlington amplifier implemented with a powertension converter to control the electric power used by the woofer. The woofer box has internal dimensions of $180 \times 180 \times 210 \mathrm{~mm}$; the walls and the cover sheet are made of a $19 \mathrm{~mm}$ MDF boards. For the design of standingwave devices as well as traveling waves, the first parameter that needs to be set is the length of the resonator, here chosen to be $225 \mathrm{~mm}$. Two different resonators were used. Because both the resonators are made of PVC, modification in the resonator length could be obtained by acting on the aluminum cork. The cork displacement range needs to be small, because the resonator length fixes the resonance frequencies $f$ according to:

$f=n \frac{c}{4 L}$

where $L$ is the longitudinal length of the cylinder $n$ is an odd number, $c$ is the speed of sound. Cylinders with a single open end produce only odd harmonics. When $n=1$ the cylinder works and produces fundamental harmonic.

The harmonic frequency, $f$, sets also the thermal penetration depth $\delta_{k}$ :

$\delta_{k}=\sqrt{\frac{k}{\pi f \rho c_{p}}}$
Fig. 1 Device scheme.

(a) Basic device scheme.

(b) The refrigerator discussed in

this paper

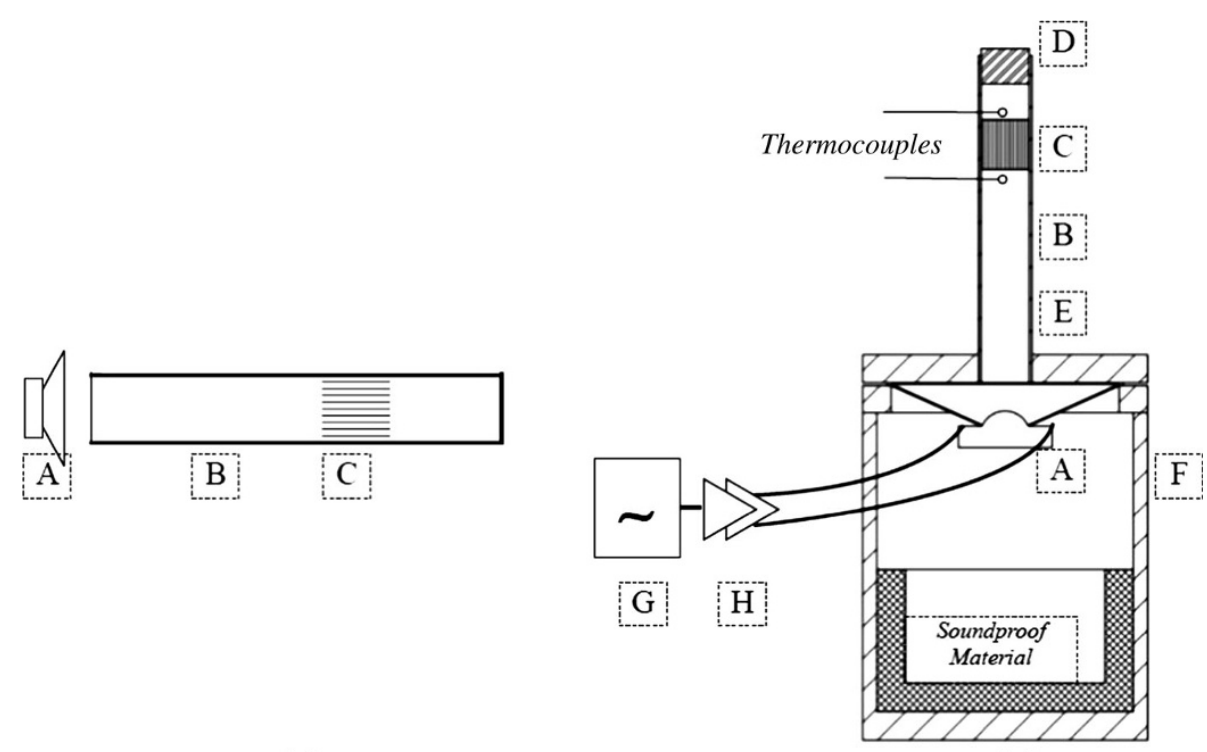

(a) 
One penetration depth corresponds to the distance traveled by heat through the working fluid in $1 \mathrm{~s}$. It depends on the fluid properties and working conditions. The spacing between the walls in the stack channels is vital for the good functioning of the refrigerator. If the spacing is too small the stack obstructs the standing-wave set up; if the stack channels are too wide, many parcels in every channel are in weak thermal contact with the stack and do not effectively transfer heat to and from it. The ideal solution, halfway between these two extremes, is described by Swift [9] and consists in a channel width of 4 thermal penetration depths.

The first step for a correct stack design is to choose a working fluid (in this case air) and fix the frequency. Fixing a frequency in standard working conditions consequently means to fix the tube length and the stack channels width. A good compromise between low frequencies (that involve long tubes) and small dimensions is to use a range of lengths from 20 up to $25 \mathrm{~cm}$ or, in terms of frequencies, from 340 up to $430 \mathrm{~Hz}$.

\section{Circuit}

In most of the available articles in thermoacoustic literature, the thermoacoustic phenomena are analyzed without discussing how sound should be generated. It is common knowledge that the easiest way to generate the sound is to use a multi-functional signal generator set up at the proper frequency. Alternatively, the signal can be generated by a computer with an electro-acoustic software and amplified through the auxiliary socket in a common home $\mathrm{Hi}-\mathrm{Fi}$ sound system. These solutions are easy to set up in a laboratory but are not effective in real applications. Multifunctional signal generation, $\mathrm{pc}$ and $\mathrm{Hi}-\mathrm{Fi}$ are designed for other purposes, furthermore the thermoacoustic refrigerators just need a single-frequency signal amplified for the woofer. For this reason, in this work, a harmonic oscillator and a signal amplifier were used. A simple circuit that creates a sinusoidal output is the Wien bridge (Fig. 2). It is based on a network made of three resistors, two capacitors, an operational amplifier and a bulb, which acts as a thermo-sensible resistor used to limit the oscillation distortions through the amplitude stabilization, as suggested by Hewlett [10]. The frequency is expressed as a function of $R$ and $C$ :

$f=\frac{1}{2 \pi R C}$

The operational amplifier needs to be well chosen as a function of the operating frequency. For frequencies below $1 \mathrm{kHz}$, suitable Op-Amp types are 107, 108, 741, 1556 and 8007 [11].

A variable resistor is used to tune the oscillator. The other two resistors in the circuit are fixed and affect the stability

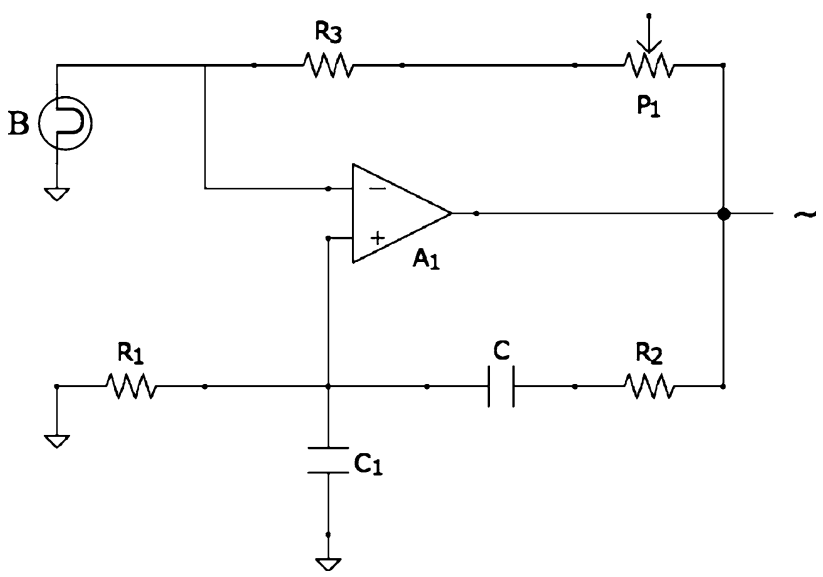

Fig. 2 Wien bridge oscillator

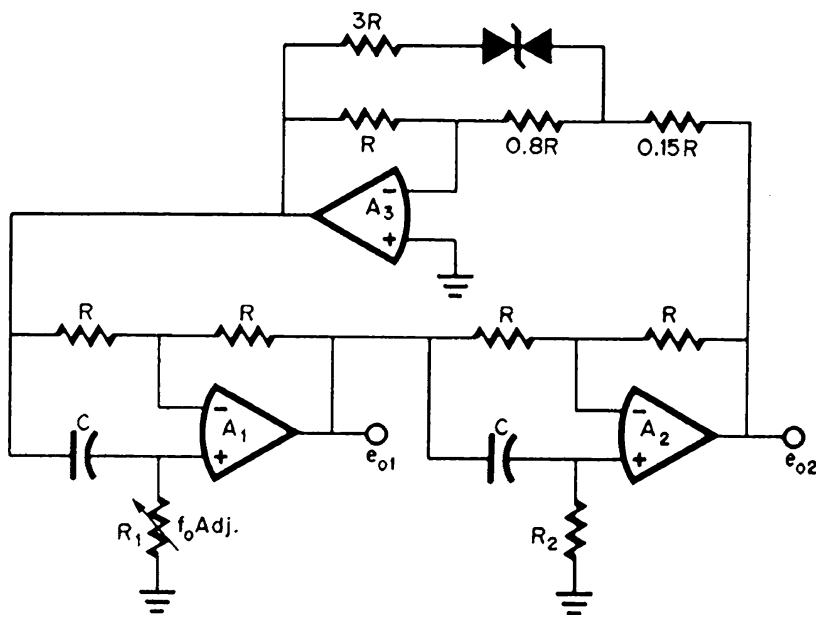

Fig. 3 Op. Amp. oscillator [12]

and the frequency range of the signal. The best use for a Wien bridge is to operate at a fixed frequency. For the Wien bridge oscillator, the frequency-determining elements also affect the critical gain of the positive feedback loop. Thus, any change in frequency made by altering these components must be followed by a precise adjustment of gain. If the signal is excessively noisy, or the frequency range is too narrow, or there are instabilities during long tests, a different oscillator should be employed. Fox example, Fig. 3 shows the oscillator used in this work, where the amplifiers $A_{1}$ and $A_{2}$ form a phase-shift circuit. This architecture allows to change the oscillation frequency without disturbing the gain magnitude of the feedback loop. The frequency equation associated with the circuit is similar to Eq. (3), but $R$ is replaced by the geometric mean value between $R_{1}$ and $R_{2}$ [12]:

$f=\frac{1}{2 \pi C \sqrt{R_{1} R_{2}}}$

The generated signal has to be amplified before reaching the woofer. Audio amplifiers can be constructed in a 
number of ways; for instance, a low-cost choice consists in using a transistor amplifier. Other amplifiers, like vacuum tube or operational amplifiers, can have larger costs, and their use is appreciably advantageous only for high-fidelity sound reproduction. The purpose of this amplifier is uniquely to increase the amplitude of a perfectly sinusoidal signal, hence there is no need to choose high-performance or multi-way amplifier. For our purpose the few parameters that need to be controlled are maximum power and, possibly, saturation.

\section{Woofer box}

In thermoacoustic literature, boxed loudspeakers or boxed woofers are mentioned, but it is necessary to specify the dimensions, shape and materials of the box. In fact, a good design can improve the woofer response at the operating frequency, reduce the sound emission of the device and decrease or eliminate the rear emission that is in phase contrast with the frontal one. For low-frequency sounds an acoustic-suspension woofer can be used. Acoustic suspension or air suspension uses the air volume sealed in the box as an elastic cushion to reinforce the oscillation of woofer diaphragm. Clearly, the volume of the box needs to be properly calculated. In order to do this, freeware programs for air-suspension boxes design like BassPc can be used. If the frequency is higher than $200 \mathrm{~Hz}$ the software can recommend unrealistically small volumes.

Besides, if the woofer is a 2-way speaker (woofer and tweeter in the same device), there is a duct that connects the bottom of the woofer (in the box) with the top, so that the air suspension cannot be used. A similar solution used for pressurized devices working with different gases is the 'gas spring system' [23]. In this case the different properties of the working fluid allow to choose this solution even with high resonance frequencies. If the rear gas volume, type and pressure can be adjusted, the gas spring can be tuned at the working frequency [13].

If the air suspension cannot be implemented, it is impossible to exploit the rear emission to enforce the front one. Moreover, if the box is not correctly designed, the rear sound may be reflected, thus interfering with the front emission. In these cases it is better to design the box to eliminate the rear emission. One possibility is to remove the box and keep the woofer in mid air (e.g., attaching it to a sort of frame); in this way, the wave coming from the bottom of the membrane is dispersed in the environment. This solution involves loud sounds coming from the device and low comfort for anybody close to the woofer. Another possibility is to try to apply the infinite-baffle model, and mount the loudspeaker in a very large sealed enclosure. This minimizes the change in the driver's resonant frequency caused by the enclosure. If it is not possible or convenient to use large volumes,one should choose a box large enough to contain the woofer, much deeper than wider and with the bottom covered by soundproof material. This solution is just an approximation of the infinite-baffle model, but for the monotone wave of a thermoacoustic device the results are satisfactory. In both the air suspension and the infinite-baffle solutions, the choice of box material and thickness is necessary. An excellent material that avoids the possibility of wall resonance is the 19-mmthick medium-density-fiberboard, or MDF, a composite wood product similar to particleboard.

Stack and tube

With air as working fluid, $\left[c_{p}=1,005 \quad \mathrm{~J} /\right.$ $\left.(\mathrm{kg} \mathrm{K}), \rho=1.205 \mathrm{~kg} / \mathrm{m}^{3}, \quad k=0.025 \mathrm{~W} /(\mathrm{m} \mathrm{K})\right]$ and a frequency range of $340-430 \mathrm{~Hz}, \delta_{k}$ ranges from 1.2 to $1.4 \times 10^{-4} \mathrm{~m}$. The spacing between the stack layers has to be approximately $4-5 \times 10^{-4} \mathrm{~m}$. Here, two different methods were used to create the stacks; in both of them a $4 \times 10^{-4} \mathrm{~m}$ nylon line is used for spacing.

The first method consists in spooling the nylon line on a $35 \mathrm{~mm}$ teflon planking in such a way to create a coil every $4 \mathrm{~mm}$. Then a film of spray glue is laid on the spooled line and a $35 \mathrm{~mm}$ photographic film is pressed on the glued line. After that, the line in excess is cut and the film is rolled to create a cylinder (Fig. 4a).

The second method consists in superimposing many microscope glass slides separated by pieces of nylon line. The slides are rectangular and have an average thickness of $1.7 \times 10^{-4} \mathrm{~m}$. The slide stack is fixed on the two lateral faces with melted EVA (ethylene-vinyl acetate). This procedure creates a parallelepiped-shaped stack that needs to fit into a circular cylinder. After the EVA consolidation, the pieces of nylon line can be taken out. The melted acetate is also used to create the cylindric frame in which the stack is set (Fig. 4b).

Two different diameter resonators have been tested. The small tube has a diameter of $22 \mathrm{~mm}$, the other $36 \mathrm{~mm}$. In both cases it is important to assure a perfect sealing of the cork: the closed end of the tube is subjected to high pressure variations and even a small escape of air can nullify the standing wave. For this reason, the corks are ripped with teflon tape. Another contact that needs to be sealed is the woofer frame surface touching the MDF sheet: for this, a $5 \mathrm{~mm}$ plastic foam gasket has been used.

The fundamental standing wave associated with resonance in air columns is visualized in Fig. 5 in terms of pressure and velocity variations in the column. A node for displacement is always an antinode for pressure and vice versa. When the air is constrained to a node, the air motion will be alternately compressed toward that point and expanded away from it, causing the pressure variation to 
Fig. 4 a Rolled film stack. b Parallel plates stack

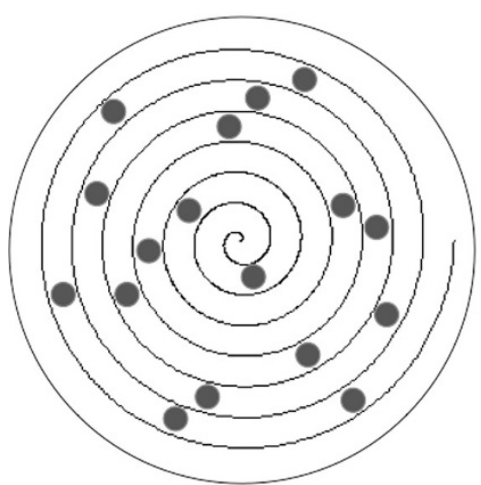

(a)

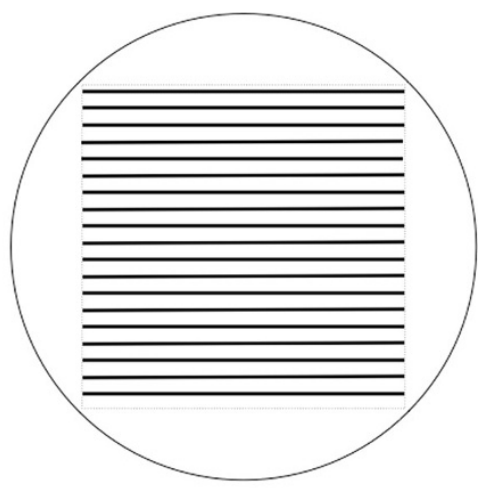

(b)

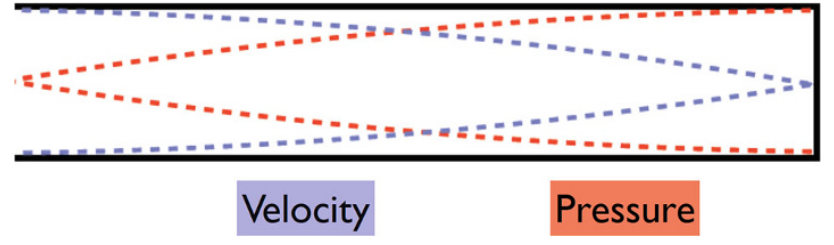

Fig. 5 Standing wave in closed end air column

reach a maximum. The fundamental harmonic of a closedend-cylinder shows that the gauge pressure variation has a maximum at the closed end and is approximately zero at the open end near the woofer (where the displacement is maximum).

\section{TA refrigerator working principles}

Once the resonance frequency of the tube is set, the device can start working. The sound coming from the woofer creates a standing wave in the tube. The air parcels in the tube start oscillating back and forth. The standing wave is associated with a stationary pressure and velocity fields as the particles are oscillating between two points, each at a different pressure. The alternation of compression and rarefaction of the gas causes the local temperature of the gas to oscillate with it, due to the adiabatic nature of sound waves. During the first half of the oscillation the particles moves and reach an high pressure area. Due to the pressure increase they shrink, and consequentially the local temperature rises. In this phase, the hot and shrunk parcels are able to transfer heat to the nearby stack wall, if the latter is colder than the gas parcels. Similarly, when the parcels oscillate back to a low-pressure area, the rarefaction of the gas causes the local temperature to drop, and if the temperature of the parcels becomes lower than that of the walls, heat is transferred from the walls to the parcels.

This alternation of compression and expansion results in a thermodynamic cycle in which the stack acts as a cold sink and a hot source in the terminal points of the oscillation of each parcel. Consequently each particle of gas pumps a little heat from the bottom of the stack to the top, from cold to hot, during each cycle of the sound wave. This effect is called "bucket brigade", to underline the contribution of every single particle cycle to the total effect. Despite the high velocities involved in the process, the thermodynamic reference cycle is the reversed Stirling cycle as reported by [22].

\section{Results}

\section{Experimental tests}

The apparatus was tested with different diameter resonators and with two different stacks. In order to compare the $22 \mathrm{~mm}$ and the $35 \mathrm{~mm}$ stacks they were both tested for $310 \mathrm{~s}$, to reach the steady-state operating condition. Two $1.5 \mathrm{~mm} T$-type thermocouples were placed at the top and bottom faces of the stacks, acquiring temperatures every $10 \mathrm{~s}$ with a sensibility of $0.1^{\circ} \mathrm{C}$. To detect which is the highest power that the woofer can run, two different phenomena must be considered. The amplification circuit is designed for amplifying music, while working with a monotone high amplitude signal is harder. In order to ensure the circuit safety, the temperature of Darlington transistors was monitored with an IR pyrometer, and the test has been stopped every time the superficial transistor temperature was higher than $90{ }^{\circ} \mathrm{C}$. To keep the system working in linear conditions, as suggested by [1], it is important to increase the power until a second harmonic is barely audible. Once the highest admissible power tolerable by all the resonators was reached, the test was started. The first test compared the different stack designs, then the best stack design was built in two diameters to compare the influence of resonator diameter on the refrigerator performance. All the tests were run fixing the working power at $30 \mathrm{~W}$ RMS. All the tube lengths were set at $225 \mathrm{~mm}$ with a respective resonating frequency of $383 \mathrm{~Hz}$. 
Table 1 Cumulative function comparison

\begin{tabular}{lllll}
\hline $\begin{array}{l}\text { Resonator and } \\
\text { stack }\end{array}$ & $\begin{array}{l}\Delta T \\
\max \\
\left({ }^{\circ} \mathrm{C}\right)\end{array}$ & $\begin{array}{l}\text { Error }_{\Delta T} \\
\left({ }^{\circ} \mathrm{C}\right)\end{array}$ & $\begin{array}{l}\text { Cumulative } \\
\text { value }\end{array}$ & $\begin{array}{l}\text { Error }_{\text {cumulative }} \\
\left({ }^{\circ} \mathrm{C}\right)\end{array}$ \\
\hline $\begin{array}{l}36 \text { mm pvc tube, } \\
\text { parallel plates } \\
\text { stack }\end{array}$ & 4.5 & 0.2 & 107 & 6.4 \\
$\begin{array}{l}36 \mathrm{~mm} \text { pvc tube, } \\
\text { rolled film stack }\end{array}$ & 6.1 & 0.2 & 148.5 & 6.4 \\
$\begin{array}{c}22 \mathrm{~mm} \text { pvc tube } \\
\text { rolled film stack }\end{array}$ & 24 & 0.2 & 688.5 & 6.4 \\
\hline
\end{tabular}

Evaluation parameter

Table 1 shows the comparison between the three different tests. The comparison is made with the assistance of a cumulative function obtained as the summation of the 32 values of $\Delta T$ for each test. This value is a time-stepwise approximation of the $t-T$ function integral, and it stands as an index of the device capability to create and maintain the temperature difference between the stack faces. Because the error that affects the measurements is $0.1^{\circ} \mathrm{C}$, the error on the $\Delta T$ is 0.2 while the one on cumulative function is $6.4{ }^{\circ} \mathrm{C}$.

\section{Test results}

The thermoacoustic refrigerator analyzed here resulted just a concept device used for demonstrating the interaction between sound and heat, but it performed the duties of a real stand-alone thermoacoustic refrigerator. From a practical point of view, during the very first start up it is necessary to tune the oscillator at the appropriate frequency, then the volume can be increased until the maximum power is reached (see previous section), then the device can start working standing alone. This thermoacoustic refrigerator complexity, despite the addition of the circuits, is still less than that of a common refrigerator; moreover, it is important to highlight that the circuit complexity will not increase in the event that the resonant-stack part is improved using different materials, geometries or gases.

Table 1 shows the $\Delta T_{\max }$ reached by every resonator that has been tested. Best results are obtained with the small diameter resonator and rolled stack, for which the $\Delta T_{\max }$ is approximately $24^{\circ} \mathrm{C}$. Such a temperature difference, compared with other simple standing-wave refrigerators in literature [1, 13, 14], shows that the self-working apparatus has worked as well as the other refrigerators.

The results with different diameter resonators or different stacks show the importance of a correct geometry and material choice. The second stack (parallel plates) was designed and built on the same fundamental parameter of

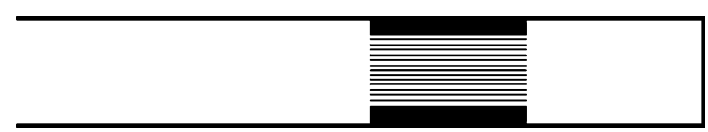

Fig. 6 Closed end tube with nozzle

the first one. The space between the plates is the same, and the wall thickness is similar. Quite surprisingly, the two stacks have led up to completely different results. In particular, the $\Delta T_{\max }$ for the parallel plates stack is about $25 \%$ less than the rolled stack. These strongly different results are caused by the difference of the effective operative areas of the two stacks. The cylindrical rolled film fits perfectly in the tube, so its whole area competes to the result; the second stack is composed of two different parts: the parallelepiped-shaped stack and the plastic cylindric frame, so the part that really operates is only the central one. In this case, two different negative effects occur: the cumulative working surface of parallel slides stack is less than the rolled stack surface; moreover the plastic frame behaves as a nozzle that can produce little variations in the resonance frequency of the tube (Fig. 6).

As previously discussed, standing-wave devices are based on the resonance phenomenon; hence little differences between the two stacks involve big variations in the thermal efficiency of the system.

More interesting is the difference between small and large diameter devices. Once is proved that the rolled stack works better than the parallel-plates one, two devices with the same kind of rolled stack but different diameters were tested. In principle, the larger the diameter (i.e. the higher the number of channels in the stack), the more the heat that can be pumped out. However, the tests show that the larger tube creates a smaller $\Delta T$. A possible explanation for this thermal behavior is that, if the acoustic power coming from the woofer is the same, the advantage of having an increased number of channels is completely nullified by the lower specific impedance inherent to the larger duct. If the specific impedance decreases, even the $\Delta p$ in every particle cycle decreases, thus creating a lower $\Delta T$ between the opposite faces of the stack. In summary, the difference between the different resonators is summarized in Table 1, the cumulative function shows the great difference and the performance boost obtained using rolled film stacks and small diameters. These results do not suggest to use small diameter resonators, but to use small diameter tubes in proximity of the stack, putting the stack between a convergent divergent tube.

Figures 7, 8 and 9 show the temperature trends during the three different tests. All the tests show that a steadystate can be reached within a time frame of $300 \mathrm{~s}$, no matter if the temperature difference established between the opposite stack faces is high or low. These results 
Fig. $736 \mathrm{~mm}$ resonator, $L=225 \mathrm{~mm}, f=383 \mathrm{~Hz}$; parallel plates stack
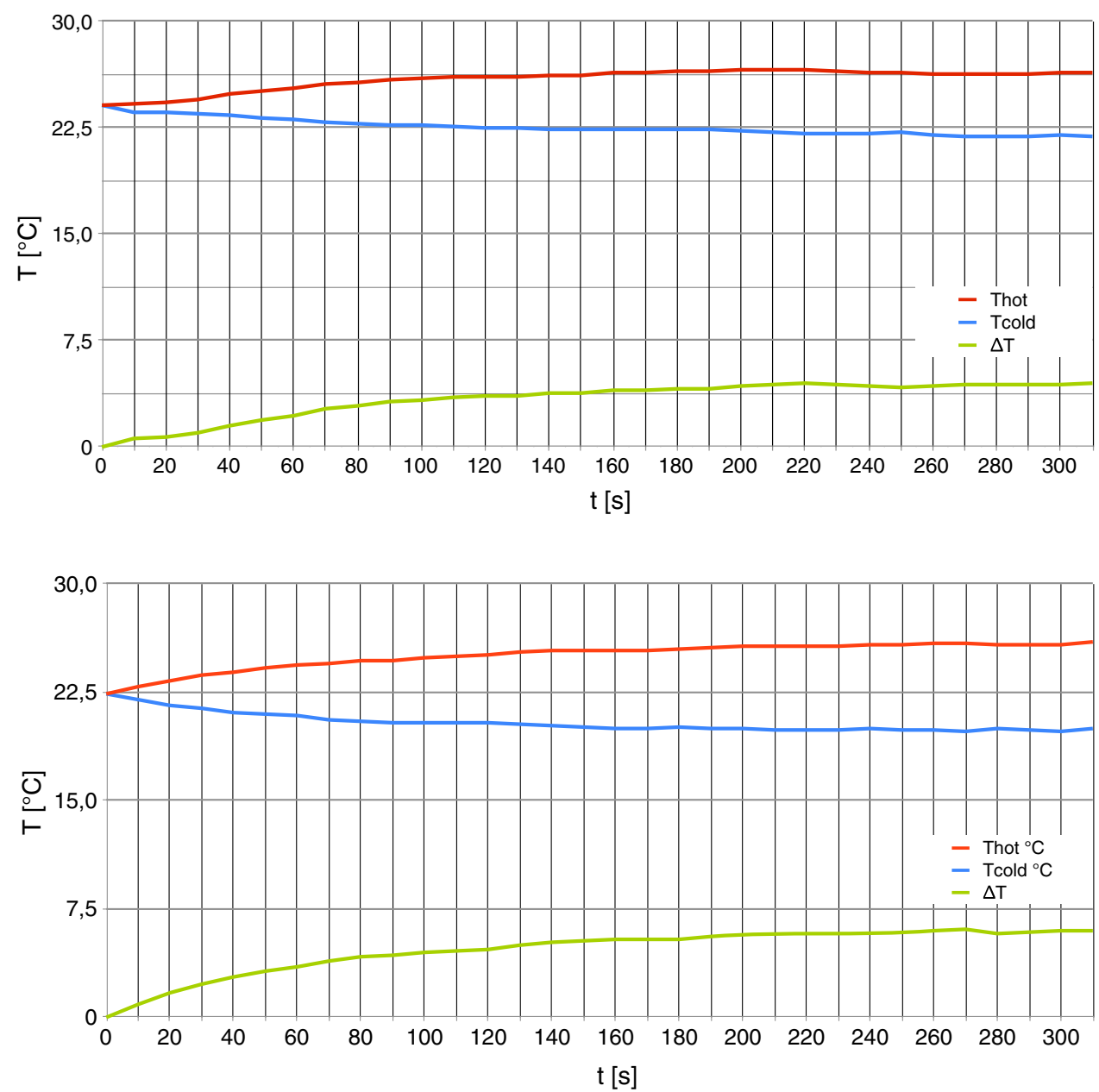

Fig. $836 \mathrm{~mm}$ resonator, $L=$ $225 \mathrm{~mm}, f=383 \mathrm{~Hz}$; rolled film stack

Fig. $922 \mathrm{~mm}$ resonator, $L=$ $225 \mathrm{~mm}, f=383 \mathrm{~Hz}$; rolled film stack

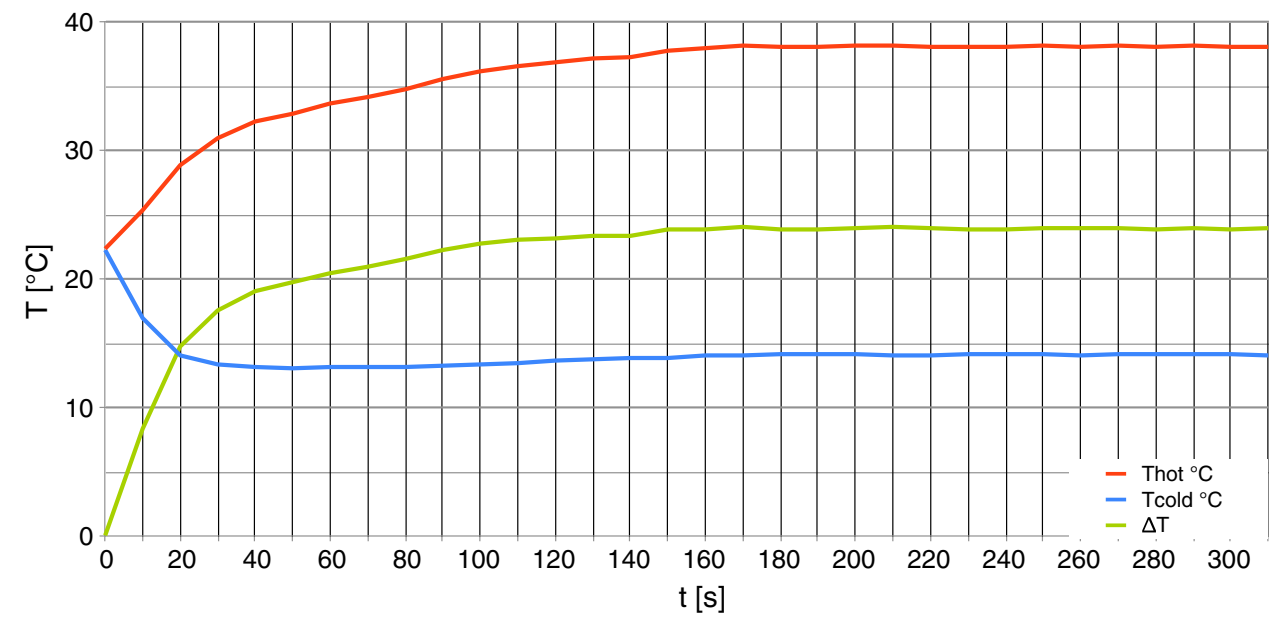

guarantee short start-up-time devices even for higher efficiency. Figures 7, 8 and 9 also display the horizontal asymptotic trend of the temperature difference curves ' $\Delta T$, in each of the cases analyzed. Such trends lead to two different considerations.
First, the asymptotic trend is a consequence of the steady-state reached by the stack: the thermoacoustic conversion allows a constant heat rate pumping from the bottom to the top of the stack. Secondly, the horizontal direction of all the temperature difference curves suggests 
that the whole device has reached a steady-state too. The heat coming from the bottom of the stack is correctly pumped out through the aluminum cork. If the device is not properly designed, the heat coming from the hot face of the stack cannot be discharged to the environment, consequently the device overheats and the temperature difference curve asymptote inclines.

\section{Conclusion}

A stand-alone standing-wave thermoacoustic refrigerator was designed, built and tested, on the basis of the RussellWeibull tabeltop device. The oscillator, the resonator and the power amplifier need to be tuned during the first start up then the device becomes self-sufficient.

Results show that the adoption of a dedicated oscillatoramplifier circuit, in place of artificial solutions (such as the use of a function generator), does not compromise the maximum $\Delta T$ achievable. Moreover, it is shown that the stack performance is influenced by the fundamental parameter $\delta_{k}$, as well as the extension of the stack surface. Temperature difference trends show that a steady-state is reached for all the cases examined. The heat coming from the upper face of the stack is dispersed in the environment through the cork, thus avoiding the need to adopt forced ventilation systems to assist heat discharge.

In addition, results suggest that, if the cross-section of the resonator is narrowed, creating a nozzle, the fundamental resonance frequency can change driving the device out of tune. It is also seen that small tubes perform better, thanks to a higher value of the specific impedance of the resonator.

Conflict of interest The authors have declared that no competing interest exists.

Author's contribution Giulio Allesina holds a master's degree in Mechanical Engineer and a PhD in "High Mechanics and Automotive Design \& Technology" discussing a final thesis on "Experimental and analytical evaluation of stratified downdraft gasifiers."His research and teaching focuses on renewable sources. He is teaching assistant and exams committee member for the following academic courses at the University of Modena and Reggio Emilia: Technical physics (thermodynamic and fluid dynamic), Thermodynamic and heat transfer, Energy management. He is the author of several peerreviewed articles published on italian and international academic Journals.

Open Access This article is distributed under the terms of the Creative Commons Attribution License which permits any use, distribution, and reproduction in any medium, provided the original author(s) and the source are credited.

\section{References}

1. Russell, D.A., Weibull, P.: Tabletop thermoacoustic refrigerator for demonstrations. Am. J. Phys. 70, 1231-1233 (2002)

2. Garrett, S., Hofler, T., Perkins, D.: Thermoacoustic refrigeration. ASHRAE J. 34, 28-36 (1992)

3. Hofler, T., Wheately, J., Swift, G., Migliori, A.: Acoustic cooling engine. US Patent no. 4722201 (1998)

4. Garrett, S.: Resource letter: Ta-1: thermoacoustic engines and refrigerators. Am. J. Phys. 72, 11-17 (2004)

5. Reid, R., Swift G.: Experiments with a flow-through thermoacoustic refrigerator. J. Acoust. Soc. Am. 108, 2835-2842 (2000)

6. Landsberg, P.: Cool sounds. Nature (London), 623-624 (1998)

7. Kirkconnell, C.S., Pruitt, G.R., Price, K.D.: Pulse tube cooler with internal mems flow controller. US Patent no 7263838 (2007)

8. Swift, G.: Thermoacoustic natural gas liquefier. In: Natural gas conference, Houston, TX (1997)

9. Swift, G.: Thermoacoustic engines and refrigerators. Phys. Today 48, 22-28 (1995)

10. Hewlett, W.: Modified wien-bridge oscillator. US Patent no. 2583649 (1952)

11. Berlin, H.: La progettazione dei circuiti amplificatori operazionali con esperimenti. Jackson editrice (1979)

12. Graeme, J.G.: Applications of Operational Amplifiers: Third Generation Techniques. BB electronics series (1973)

13. Tijani, M.: Loudspeaker-driven thermo-acoustic refrigeration. $\mathrm{PhD}$ thesis (2001)

14. Newman, J., Cariste, B., Queiruga, A., Davis, I., Plotnik, B., Gordon, M., Martin, S.S.: Thermoacoustic refrigeration. GSET Res. J. (2006)

15. Hariharana, N.M., Sivashanmugama, P., Kasthurirenganb, S.: Experimental investigation of a thermoacoustic refrigerator driven by a standing wave twin thermoacoustic prime mover. Int. J. Refrig. 36(8), 2420-2425 (2013)

16. Chen, M., Ju, Y.M.: Design and experimental investigations on a small scale traveling wave thermoacoustic engine. Cryogenics 54, 10-15 (2013)

17. Wu, Z., Dai, W., Man,, M., Luo, E.: A solar-powered travelingwave thermoacoustic electricity generator. Sol. Energy. Mater. Sol. Cells 86(9), 2376-2382 (2012)

18. Ghorbanian, K., Karimi, M.: Design and optimization of a heat driven thermoacoustic refrigerator. Appl. Thermal Eng. 62(2), 653-661 (2014)

19. Bansal, P., Vineyard, E., Abdelaziz, O.: Status of not-in-kind refrigeration technologies for household space conditioning, water heating and food refrigeration. Int. J. Sustain. Built Environ. 1(1), 85-101 (2012)

20. Konaina, T., Yassen, N.: Thermoacoustic solar cooling for domestic usage sizing software. Energy Proc. 18:119-130 (2012)

21. Xu, J., Liu, B., Zhao, D.: Numerical simulating for turbulent heat transfer of structural improved thermoacoustic cooler. Proc. Eng. 29, 2177-2181 (2012)

22. Yazaki, T., Biwa, T.,Tominaga, A.: A pistonless Stirling cooler. Appl.. Phys.. Lett.. 80(1), 157-159 (2002)

23. Tijani, M.E.H., Zeegers, J.C.H., de Waele, A.T.A.M.: A gasspring system for optimizing loudspeakers in thermoacoustic refrigerators. J. Appl. Phys. 92(4), 2159 (2002) 